\title{
The Rheumatoid Hand: A Predictable Disease With Unpredictable Surgical Practice Patterns
}

\author{
AMY K. ALDERMAN, ${ }^{1}$ KEVIN C. CHUNG, ${ }^{2}$ SONYA DEMONNER, ${ }^{3}$ SANDRA V. SPILSON, ${ }^{4}$ \\ AND RODNEY A. HAYWARD ${ }^{5}$
}

Objectives. To evaluate variation in fusion, arthroplasty, and tenosynovectomy rates among rheumatoid arthritis (RA) patients across states; to evaluate associations between surgery rates and the density of hand surgeons; and to evaluate differences in treatment by sex of the patient.

Methods. Data were obtained from the 1996 and 1997 Healthcare Cost and Utilization Project database. The procedure codes for fusion, arthroplasty, and tenosynovectomy were matched to patients with the diagnostic code of RA, which provided the total number of procedures performed in each state. The smoothed estimates of the RA population for each state were derived from age/sex strata in the 1995 US census using age/sex-adjusted RA prevalence data from the Third National Health and Nutrition Examination Survey. The number of hand surgeons was from the 1996 American Society for Surgery of the Hand.

Results. Procedure rates across states varied from 9-fold to 12-fold for all 3 procedures. The rates of the reconstructive procedures-fusion and arthroplasty—were highly correlated in each state, but these 2 procedures were only moderately correlated with tenosynovectomy. Surgeon density and procedure rates were minimally correlated. Procedure rates differed by patient sex, with significantly more arthroplasty and fusion procedures performed in women. More tenosynovectomy procedures were performed in men, and they were also performed at a younger age in men.

Conclusions. Significant large area variations are present in the surgical management of the rheumatoid hand, but the correlations between reconstructive and early intervention procedures are modest. These rate differences are not explained by the number of hand surgeons, disease prevalence, or demographic composition of the states. However, men are more likely to receive more aggressive early surgical interventions, and women are more likely to receive end-stage reconstructive surgery.

KEY WORDS. Rheumatoid arthritis; Large area variations; Tenosynovectomy; Arthroplasty; Arthrodesis.

\section{INTRODUCTION}

Rheumatoid arthritis (RA) is a debilitating and progressive disease, affecting an estimated 2.1 million Americans (1).

Supported by grants from the Robert Wood Johnson Foundation and the American Society for Surgery of the Hand.

${ }^{1}$ Amy K. Alderman, MD: The University of Michigan and the University of Michigan Medical Center, Ann Arbor, Michigan; ${ }^{2}$ Kevin C. Chung, MD, MS: The University of Michigan Hand Center, Ann Arbor, Michigan; ${ }^{3}$ Sonya DeMonner, MPH: The University of Michigan, Ann Arbor, Michigan; ${ }^{4}$ Sandra V. Spilson, MPH: The University of Michigan Medical Center, Ann Arbor, Michigan; ${ }^{5}$ Rodney A. Hayward, MD: Ann Arbor VA Health Care System and The University of Michigan, Ann Arbor, Michigan.

Address correspondence to Kevin C. Chung, MD, MS, Section of Plastic Surgery, University of Michigan Medical Center, 2130 Taubman Center, 1500 East Medical Center Drive, Ann Arbor, MI 48109-0340. E-mail: kecchung@umich. edu.

Submitted for publication August 7, 2001; accepted in revised form February 12, 2002.
Although RA is a systemic disease extending beyond the musculoskeletal system, more than $70 \%$ of RA patients have some form of hand disability (2). The disease often progresses to crippling hand deformities despite aggressive medical treatment, and few patients ever achieve disease control (3).

The role of surgery in the rheumatoid hand has been controversial, with rheumatologists and surgeons debating the efficacy of surgical treatments. This controversy may result in uncertainty in patient management, which can lead to large variation in surgical practice across the country (4). Furthermore, given the uncertainty of treatment efficacy cited in the medical literature, the rheumatoid population may be particularly vulnerable to treatment biases, with influences ranging from patient sex to the density of health care providers (5-11).

The purpose of our research was to use a large, population-based sample to evaluate surgical practice patterns of the rheumatoid hand in the US. Specifically, we evaluated 3 surgical procedure rates across the country, looking for evidence of geographic variation in practice patterns. We 
also evaluated aggressiveness of care between men and women, comparing the rates of end-stage reconstructive procedures, arthroplasty and arthrodesis, with the rates of prophylactic tenosynovectomy. In addition, the procedure rates were compared with the density of surgeons, assessing the possibility of physician-induced demand.

\section{MATERIALS AND METHODS}

Database characteristics. We obtained data from the 1996 and 1997 Healthcare Cost and Utilization Project (HCUP)-3 Nationwide Inpatient Sample Release 5 and 6. This national database is sponsored by the Agency for Healthcare Research and Quality, formerly the Agency for Health Care Policy and Research. The project involves a partnership between federal and state government and industry to collect national health care data for use in research and policy planning. For each year, the database contains a $20 \%$ stratified sample of more than 6.5 million hospital discharges from more than 906 hospitals, including both academic and private institutions. The Veterans Healthcare Administration system and ambulatory surgery cases are excluded from the database. The database uses a stratified probability sample of hospitals, with sampling probabilities proportional to the number of hospitals in each stratum. HCUP is one of a limited number of resources that contains information on hospital discharges for all payers, including Medicare, Medicaid, private insurance, and the uninsured.

Using the HCUP database allowed us to evaluate the incidence of arthroplasty, arthrodesis, and tenosynovectomy procedures in RA patients and to examine associations with the following variables: patient demographics, payment information, and characteristics of the hospitals, such as teaching status and geographic location. In addition, the database has internal weighting factors that generate state and national incidence estimates for the procedures.

Study population. Our target population, patients with RA, was identified using the International Classification of Diseases, Ninth Revision, Clinical Modification (ICD-9CM) diagnostic code 714.0. Patients were then selected who had undergone one or more ICD-9 procedures specified for the hand: arthrodesis (81.25-81.28), arthroplasty (81.71-81.75), and tenosynovectomy (82.33-83.42). The arthrodesis codes include carporadial, metacarpocarpal, metacarpophalangeal, and interphalangeal fusions. The arthroplasty codes include reconstruction with and without implants of the metacarpophalangeal, interphalangeal, carpocarpal, and carpometacarpal joints. The tenosynovectomy code was a general code for all tenosynovectomy procedures of the hand.

In 1996, 19 states were sampled, and in 1997, 22 states were sampled. For evaluation of procedure rates across states, we limited the study population to the 19 states sampled from both years. This included the following states: Arizona, California, Colorado, Connecticut, Florida, Illinois, Iowa, Kansas, Massachusetts, Maryland, Missouri, New Jersey, New York, Oregon, Pennsylvania, South Carolina, Tennessee, Washington, Wisconsin. To compare pro- cedure rates across states, we estimated the number of people with RA in each state. We used the 1995 US census to obtain the total number of men and women in each of the sampled states for 3 different age categories (17-24 years, 25-64 years, and $\geq 65$ years), and the prevalence of RA within each age/sex stratum was obtained from the Third National Health and Nutrition Examination Survey (NHANES-III), which is a national database of patients' self-assessments of health. For each state, we multiplied the population in each of the 6 age/sex categories by the smoothed age/sex-specific prevalence of RA to obtain our age/sex-adjusted estimates of the RA population. The smoothed estimates of RA prevalence use fitted estimates derived from a regression model to increase the precision of the estimates and decrease the effects of outliers and random variation. For example, Arizona had 137,424 women between the ages of 17 and 24. This figure was multiplied by 0.003 (the prevalence of RA in women in this age group) to give an estimated 412 women with RA aged 17-24. The adjusted estimates controlled for the increased prevalence of RA in women and older age groups. We limited all analyses to whites, because nonwhites have low RA prevalence rates and account for a minority of the NHANES-III sample. Therefore, the age/sex-adjusted RA prevalence rates could not be accurately estimated.

The number of hand surgeons in each state was obtained from the 1996-1997 American Society for Surgery of the Hand (ASSH) active membership registry, which includes both orthopedic and plastic hand surgeons and excludes retired members. We felt that this organization would give us the best representation of surgeons who trained in and are practicing hand surgery and who have a special expertise in complicated hand reconstruction for the rheumatoid population. The density of surgeons per state was derived by dividing the total number of surgeons in a state by that state's total population.

Ideally, those members of the RA population who have already had surgery would be removed from the denominator. The calculation of a surgery rate is generally done only once. However, we did not have this information and were, therefore, unable to make this adjustment. Still, the overall rate of these surgeries is so low that this adjustment would have a minimal effect on our results and would tend to result in underestimates of practice variation.

Data analysis. The calculations for surgery rates in each state were generated by dividing the total number of procedures for men and women by the age/sex-adjusted RA population. For example, California, in which 170 arthroplasties were performed, has an RA population of 126,249, which equates to a procedure rate of 13.5 arthroplasties per 10,000 RA patients for 1996 and 1997, or an annual rate of 6.7 per $10,000 \mathrm{RA}$ patients. Logistic regression provided a comparison of the probability of having a procedure across the 19 sampled states, analyzing the extent of state-to-state variation. In this regression, the state was the predictor variable and receipt of the procedure was the outcome variable. For this analysis, California was chosen as the reference group to which all other states were compared, because it was the most populous state, and because 


\begin{tabular}{|c|c|c|c|c|}
\hline & \multicolumn{2}{|c|}{ Procedure rate $(95 \% \mathrm{CI})^{*}$} & \multirow[b]{2}{*}{ Odds ratiot } & \multirow[b]{2}{*}{$\boldsymbol{P}$} \\
\hline & Men & Women & & \\
\hline Arthroplasty & $2.3(1.7-3.0)$ & $7.7(7.0-8.5)$ & 3.36 & 0.001 \\
\hline Arthrodesis & $2.6(2.0-3.3)$ & $3.4(2.9-3.9)$ & 1.30 & 0.009 \\
\hline Tenosynovectomy & $13.9(12.4-15.4)$ & $5.8(5.2-6.5)$ & 0.42 & 0.001 \\
\hline
\end{tabular}

we had no reason to suspect any particular state to display significant variations in practice patterns compared with other states.

To evaluate significant differences in procedure rates between men and women, we performed chi-square analysis and generated odds ratios (ORs) and 95\% confidence intervals (95\% CI). Student's $t$-test was performed to compare the difference in mean age between men and women for each procedure. Other analyses included Pearson's correlation coefficient to measure the relationship between each procedure's incidence rate and the density of hand surgeons across states. For the correlation between arthrodesis and the other variables (tenosynovectomy, arthroplasty, and surgeon), Oregon was an outlier because it had a rate of arthrodesis that was out of proportion to the other states. Thus, this state was omitted from the analysis. All analyses were performed with STATA version 6.0 (Stata Corporation, College Station, TX) and SAS version 6.12 (SAS Institute, Cary, NC) statistical software packages, and statistical significance was set at two-sided $P<0.05$.

\section{RESULTS}

Of the procedures we evaluated, Medicare covered 44$64 \%$ of the patients, private health insurance covered $29-$ $38 \%$, and fewer than $7 \%$ of the patients were uninsured. Of the 772 hospitals included in the sample population, 427 (55\%) performed at least 1 arthroplasty, $214(28 \%)$ performed at least 1 arthrodesis, and 601 (78\%) performed at least 1 tenosynovectomy. The distribution of the procedures was almost evenly split between reconstructive and prophylactic procedures: $52 \%$ versus $48 \%$, respectively. Of the reconstructive procedures, 34\% were arthroplasty and $18 \%$ were arthrodesis techniques. The length of hospital stay was 1 day for $50 \%$ of fusions, $50 \%$ of arthroplasties, and $69 \%$ of tenosynovectomies; the remainder of the cases generally stayed 5 or fewer days. The percentage of procedures performed in the urban teaching and nonteaching hospitals was evenly split, with urban hospitals accounting for approximately $90 \%$ of the procedures performed. Patient demographics were similar across the 3 procedures, except for age and sex.

Table 1 illustrates procedure rates for men compared with women. Women outnumbered men in arthroplasty (7.7 versus 2.3 , respectively; $\mathrm{OR}=3.36, P<0.001$ ) and in arthrodesis procedures (3.4 versus 2.6, respectively; OR = $1.3, P=0.009)$. However, women were less likely to receive tenosynovectomy procedures (5.8 versus 13.9 , respectively; $\mathrm{OR}=0.42, P<0.001)$. In addition, tenosyno- vectomy procedures were associated with a significantly younger age at treatment for men compared with women (54 versus 59 years, respectively; $P<0.001$ ).

Table 2 displays the annual rates for each procedure along with the $95 \%$ CIs for each of the sampled states. Large variations in procedure rates across the 19 states were found. These variations were highly significant for all 3 procedures $(P<0.0001)$, with the difference in rates between the highest and lowest states varying by a factor of 9.4 in arthroplasty, 11.7 in arthrodesis, and 9.5 in tenosynovectomy procedures.

The Pearson correlation matrix of procedure rates and density of surgeons by state is seen in Table 3. The evaluation of one procedure rate with another revealed a strong positive correlation between the 2 reconstructive procedures, arthroplasty and arthrodesis $(\mathrm{r}=0.80)$. This relationship is visually displayed in Figure 1. However, tenosynovectomy rates were only modestly correlated with arthroplasty and arthrodesis rates $(r=0.20$ and 0.23 , respectively). Therefore, states that perform more reconstructive procedures are only slightly more likely to perform prophylactic procedures. Each state's density of hand

Table 2. Annual rates of arthroplasty, arthrodesis, and tenosynovectomy procedures in the sampled states*

\begin{tabular}{|lccc|}
\hline State & Arthroplasty & Arthrodesis & Tenosynovectomy \\
\hline AZ & $4.1(2.5-6.5)$ & $2.0(0.9-3.7)$ & $14.8(11.5-18.7)$ \\
CA & $6.7(5.8-7.8)$ & $3.2(2.6-4.0)$ & $10.9(9.6-12.2)$ \\
CO & $2.9(1.5-5.1)$ & $1.7(0.7-3.5)$ & $5.8(3.8-8.7)$ \\
CT+ & - & - & $12.2(9.1-16.1)$ \\
FL & $6.7(5.5-8.0)$ & $2.2(1.5-3.0)$ & $7.7(6.4-9.1)$ \\
IL & $3.2(2.3-4.3)$ & $2.0(1.3-3.0)$ & $6.4(5.1-8.0)$ \\
IA & $10.3(7.4-13.9)$ & $3.0(1.6-5.3)$ & $2.0(0.9-4.0)$ \\
KS & $6.4(4.0-9.8)$ & $2.2(0.9-4.4)$ & $2.8(1.3-5.2)$ \\
MA & $7.1(5.4-9.2)$ & $2.7(1.7-4.1)$ & $4.4(3.2-6.0)$ \\
MD & $7.8(5.5-10.6)$ & $4.3(2.7-6.5)$ & $10.8(8.1-14.1)$ \\
MO & $4.9(3.4-6.9)$ & $2.0(1.1-3.3)$ & $6.7(4.9-9.0)$ \\
NJ & $4.5(3.2-6.1)$ & $0.9(0.4-1.8)$ & $9.9(7.9-12.2)$ \\
NY & $4.8(3.8-5.8)$ & $2.4(1.7-3.2)$ & $4.1(3.3-5.1)$ \\
OR & $5.9(3.8-8.7)$ & $10.5(7.6-14.1)$ & $16.1(12.5-20.5)$ \\
PA & $13.2(11.5-15.1)$ & $8.0(6.7-9.5)$ & $12.6(11.0-14.4)$ \\
SC & $1.4(0.5-3.2)$ & $1.4(0.5-3.2)$ & $1.7(0.6-3.6)$ \\
TN & $2.6(1.5-4.2)$ & $2.6(1.5-4.2)$ & $6.9(5.0-9.3)$ \\
WA & $2.2(1.2-3.6)$ & $3.3(2.0-5.0)$ & $3.0(1.8-4.6)$ \\
WI & $11.5(9.1-14.4)$ & $4.2(2.8-6.1)$ & $11.1(8.7-13.9)$ \\
\hline * Annual procedure rates (95\% confidence interval) were calcu- \\
lated per 10,000 rheumatoid arthritis patients. \\
t Connecticut did not report any arthroplasty or arthrodesis proce- \\
dures. & & & \\
\hline
\end{tabular}




\begin{tabular}{|lccc|}
\hline \multicolumn{4}{|c|}{ Table 3. Associations between procedure rates and surgeon density by state* } \\
\hline & Arthrodesis & Tenosynovectomy & Surgeon density \\
\hline Arthroplasty & 0.80 & 0.20 & -0.37 \\
Arthrodesis & - & 0.23 & -0.18 \\
Tenosynovectomy & - & -0.12 \\
\hline * Data are Pearson's correlation coefficients. Surgeon density was calculated from the total number of \\
hand surgeons in each state divided by that state's population. \\
\hline
\end{tabular}

surgeons was then compared with the different procedure rates. There was no evidence that greater surgeon density was associated with greater arthroplasty, arthrodesis, or tenosynovectomy rates, and the trend actually went in the opposite direction $(\mathrm{r}=-0.37,-0.18$, and -0.12 , respectively).

\section{DISCUSSION}

Rheumatoid arthritis is a crippling systemic disease that affects an estimated $1 \%$ of the US population at the prime of their lives (1). Treatment options are limited, with no cure available. Medical management is aimed at limiting synovial proliferation and slowing the progression of joint destruction. Surgical options for hand deformities are also limited, defined as either reconstructive (arthrodesis or arthroplasty) or prophylactic (tenosynovectomy). Unfortunately, there is little information available on these procedures from rigorous outcomes research or randomized clinical trials, forcing physicians to rely on uncontrolled case series for clinical decision making.

Although RA differentially affects women, one must still consider the possibility of differences in health care delivery based on patient sex. Many studies have aroused concern regarding underutilization of surgical interventions in women, such as coronary revascularization, renal transplantation, and hip or knee arthroscopy (5-8). In our analysis, women were 3.4 times more likely than men to receive arthroplasty procedures and 1.3 times more likely to receive arthrodesis procedures compared with men. However, men were 2.4 times more likely than women to receive tenosynovectomy procedures. Conclusions from these results are limited due to a lack of information regarding the patients' disease severity and willingness to undergo surgery. Because men have higher rates of the prophylactic procedure-tenosynovectomy-and re-

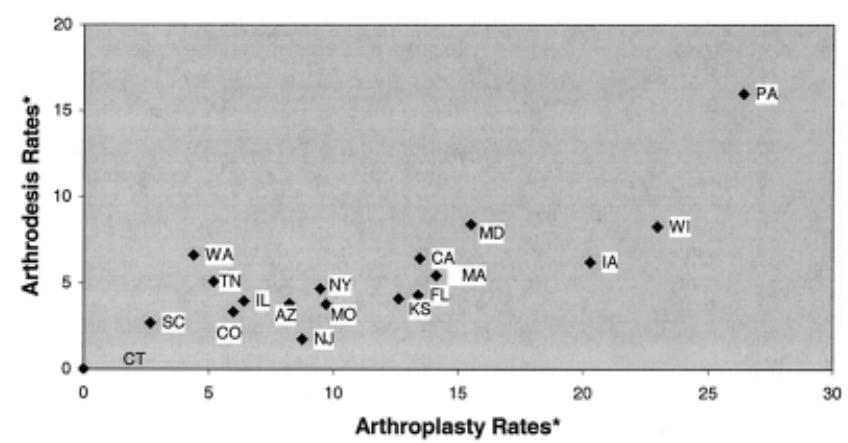

Figure 1. Correlation between rates of arthroplasty and arthrodesis by state. * Arthroplasty and arthrodesis rates are per 10,000 rheumatoid arthritis patients. ceive this procedure at a younger age than women, the question must be raised whether men are receiving more aggressive care than women. In addition, variations in procedure rates between the sexes may be the result of differences in disease manifestations, patient preferences, or physician biases and deserves further investigation.

The literature regarding treatment of the rheumatoid hand is controversial, with the efficacy of prophylactic tenosynovectomy often at the crux of the debate $(9,12-14)$. Surgeons complain of exclusion from RA management teams, as they are often called on as a source of last resort when all else fails (15). In contrast, rheumatologists portray synovectomy procedures as delaying strategies with minimal long-term efficacy (16). Many rheumatologists define the role of surgery as a salvage technique and are reluctant to consider surgery in the early stages of the disease (17). Our results indicate that some physicians believe in the efficacy of prophylactic procedures in the RA hand, as tenosynovectomy accounted for $48 \%$ of the procedures studied. This is surprising considering the lack of confidence in the rheumatology literature regarding prophylactic procedures on RA hands (16). Furthermore, advances in technology have improved arthroplasty outcomes $(18,19)$, which may alleviate some of the pressure on surgeons to intervene early with prophylactic procedures on functional hands. However, we do not know if surgeons are performing prophylactic procedures at higher or lower rates than in the past, given the changes in medical management, arthroplasty materials, and referral patterns.

Variations in practice patterns can arise when multiple clinical options are available, particularly when there is uncertainty about optimal management. For example, greater geographic variation exists with hysterectomy than with colectomy rates, due to greater treatment options for uterine fibroids and dysfunctional bleeding (4). Treatments considered to be the universal standard usually have limited practice pattern variations. Studying practice patterns is important for delineating clinical conditions for which intervention decisions may need more rigorous evaluation. Although many factors can contribute to variations, such as differences in illness rates, environmental conditions, patient preferences, access to care, and economic incentives, a large percentage of practice variations can be explained by disagreement among physicians regarding appropriate therapy (4). Generally, physicians lack consensus in patient management when treatment outcomes are poorly defined (4). The significant variation in procedure rates across states in our analyses was not sur- 
prising, given the lack of consensus in the literature regarding the treatment of the rheumatoid hand. We expected to find less variation in the rates of end-stage procedures because the literature appears less controversial in these areas. However, we found a higher than expected positive correlation between arthroplasty and arthrodesis procedures within each state. Thus, a state likely to perform one type of end-stage procedure was also likely to perform the other type. The reason for this correlationpatient preference, disease manifestations, or physician preference-cannot be elucidated from our data. However, these procedures are often used in combination to maximize stability and function, which may account for some of the correlation. For example, proximal interphalangeal fusions are combined with metacarpophalangeal arthroplasties to correct for swan neck deformities. Nevertheless, our study clearly demonstrates that one of the most influential factors in the treatment of the rheumatoid hand is the state in which the patient lives. Therefore, we now need to determine the reasons behind such wide variation in treatment.

From a health policy perspective, concern exists regarding the cost of specialized care, including hand surgery. In general, the US has an overabundance of specialized physicians, contributing to the escalating costs of health care (20). Physicians are believed to sometimes induce the demand for their own services; therefore, one attempt to control healthcare costs is to control the number of specialty providers (21). In Canada, health policy experts attribute practice pattern variations to physician density, believing that physicians induce demand for their services, which increases health care costs. Plans for restructuring the fee schedules of specialty physicians and relocating specialists to rural locations have been discussed to unify practice patterns. However, an orthopedic study of knee replacement rates showed surgery rates and density of surgeons to be unrelated, arguing against the restructuring efforts (22). Similar to the Canadian study, we found no evidence that specialized surgeons are inducing demand for their services. We found no significant relationship between the density of hand surgeons in a state and the rate of hand procedures in RA patients. Answering the question of specialty physician-induced demand is challenging and may be better approached at the county rather than the state level. However, the database does not provide zip codes, so the possibility of physician-induced demand at the county or hospital level cannot be evaluated. In addition, because the HCUP database does not identify the provider, we had no means of identifying surgeons performing these procedures who were not ASSH members. However, rheumatoid hand surgery is very complex and, according to our data, $90 \%$ of the procedures were performed in urban areas with available ASSH surgeons. Therefore, we believe that our sample of ASSH members probably reflects the general membership of physicians who care for these patients.

Although using a national database aids in the generalizability of the study results, limitations still exist. For example, the procedure rates for the sampled states were probably underestimated, because we were unable to account for patients in the population already treated by surgery and patients who had received outpatient surgery (although the popularity of ambulatory surgery was at its infancy at this time). Our study results were also limited to the white patient population due to our limited sample size of nonwhites. However, white people represent $92 \%$ of RA patients in this country so we believe that our sample adequately represented the study population (23). Lastly, databases are susceptible to errors in coding, which may underestimate or overestimate procedure rates. RA is difficult to diagnose, and patients with inflammatory arthritis may be incorrectly diagnosed as having RA. However, we used the most accurate method available to define the RA population, which was through the use of ICD9-CM diagnostic codes.

In conclusion, the surgical treatment of the rheumatoid hand is a complex multidimensional process with limited evidence-based research and limited agreement among physicians as to appropriate treatment modalities. Not surprisingly, we found significant variations in physician practice patterns on a national level, influenced both by the state in which the procedure was performed and the patient's sex, but not by the density of hand surgeons. Our future research initiatives will investigate whether this variation represents differences in physician behavior or differences in patients' wants or needs.

\section{REFERENCES}

1. Arthritis Foundation Web site. Accessed Jan 23, 2001. URL: www.arthritis.org/conditions/diseasecenter/ra.asp.

2. De la Mata Llord J, Palacios Carvajal J. Rheumatoid arthritis: are outcomes better with medical or surgical management? [editorial]. Orthop 1998;21:1085-6.

3. Benson WB, Bensen W, Adachi JD. Back to the future: the pyramids of rheumatoid arthritis. J Rheumatology 1997;24: 1023-30.

4. Keller RB, Soule DN, Wennberg JE, Hanley DF. Dealing with geographic variations in the use of hospitals. J Bone Joint Surg Am 1990;72:1286-93.

5. Gijsbers van Wijk CMT, van Vliet KP, Kolk AM. Gender perspectives and quality of care: towards appropriate and adequate health care for women. Soc Sci Med 1996;43:70720.

6. Weitzman S, Cooper L, Chambless L, Rosamond W, Clegg L, Marcucci G, et al. Gender, racial and geographic differences in the performance of cardiac diagnostic and therapeutic procedures for hospitalized acute myocardial infarction in four states. Am J Cardiol 1997;79:722-6.

7. Bloembergen WE, Mauger EA, Wolfe RA, Port FK. Association of gender and access to cadaveric renal transplantation. Am J Kidney Dis 1997;30:733-8.

8. Hawker GA, Wright JG, Coyte PC, Williams JI, Harvey B, Glazier R, et al. Differences between men and women in the rate of use of hip and knee arthroscopy. N Engl J Med 2000; 342:1016-22.

9. McEwen C. Multicenter evaluation of synovectomy in the treatment of rheumatoid arthritis: report of results at the end of five years. J Rheumatol 1988;15:764-9.

10. Tolat AR, Stanley JK, Evans RA. Flexor tenosynovectomy and tenolysis in longstanding rheumatoid arthritis. J Hand Surg [Br] 1996;21:538-43.

11. Grumback K, Bodenheimer T. Mechanisms for controlling costs. JAMA 1995;273:1223-30. 
12. Gariepy R, Demers R, Laurin CA. The prophylactic effect of synovectomy of the knee in rheumatoid arthritis. Can Med Assoc J 1966;94:1349-52.

13. McEwen C. Early synovectomy in the treatment of rheumatoid arthritis. N Engl J Med 1969;279:420-2.

14. Mongan ES, Boger WM, Gilliland BC, Meyerowitz S. Synovectomy in rheumatoid arthritis: a retrospective study. Arthritis Rheum 1970;13:761-8.

15. Nalebuff EA. Rheumatoid hand surgery: update. J Hand Surg [Am] 1983;8:678-82.

16. Neustadt DH. Evaluation of synovectomy in rheumatoid arthritis. J Rheumatol 1988;15:1874.

17. Bird H. Current management of rheumatoid arthritis. Br J Hosp Med 1986;35:374-81.

18. Block JA. Prevalence of arthroplasty during the course of rheumatoid arthritis. Lancet 1998;352:1086.
19. Chung KC, Kowalski CP, Kim HM. Patient outcomes following Swanson silastic metacarpophalangeal joint arthroplasty in the rheumatoid hand: a systematic overview. J Rheumatol 2000;27:1395-1402.

20. Grumback K, Bodenheimer T. The organization of health care. JAMA 1995;273:3-10.

21. Evans RG. Supplier-induced demand: some empirical evidence and implications. In: Perlman M, editor. Economics of health and medical care. London: MacMillan; 1974.

22. Coyte PC, Hawker G, Wright JG. Variations in knee replacement utilization rates and the supply of health professionals in Ontario, Canada. J Rheumatol 1996;23:1214-20.

23. Dugowson CE, Koepsell TD, Voigt LF, Bley L, Nelson JL, Daling JR. Rheumatoid arthritis in women: incidence rates in Group Health Cooperative, Seattle, Washington, 1987-1989. Arthritis Rheum 1991;34:1502-7. 